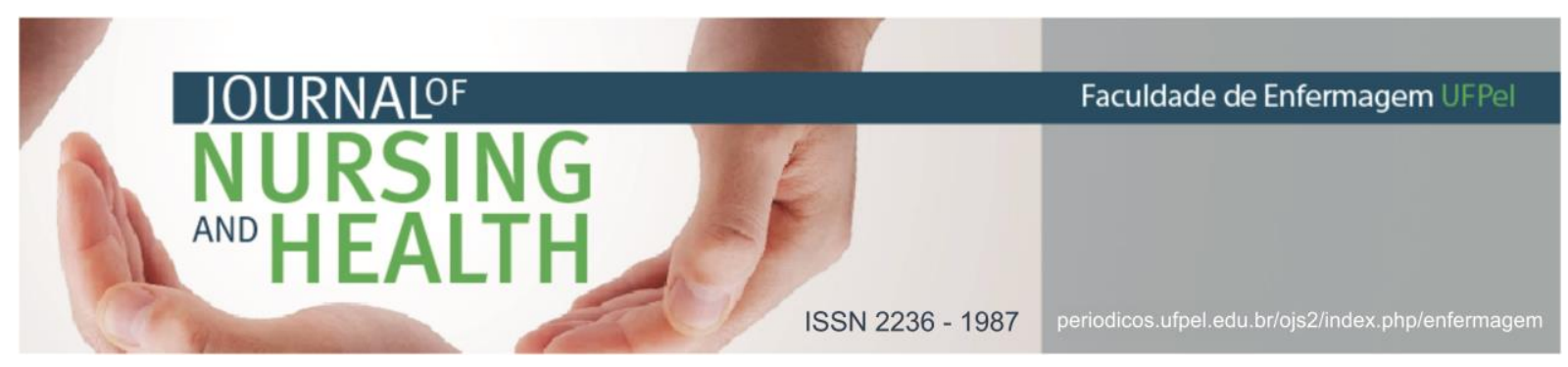

ARTIGO DE REVISÃO

\title{
Saúde mental na estratégia saúde da família: caminhos para uma assistência integral em saúde
}

\author{
Mental health in family health strategy: steps to integral assistance in health \\ Salud mental en la estrategia de salud de la familia: caminos para una asistencia \\ integral en salud
}

Eslabão, Adriane Domingues ${ }^{1}$; Santos, Elitiele Ortiz dos ${ }^{2}$; Santos, Vilma Constancia Fioravante dos ${ }^{3}$; Rigatti, Roberta ${ }^{4}$; Mello, Rita Mello de ${ }^{5}$; Schneider, Jacó Fernando ${ }^{6}$

Como citar este artigo: Eslabão AD, Santos EO, Santos VCF, Rigatti R, Mello RM, Schneider JF. Saúde mental na estratégia saúde da família: caminhos para uma assistência integral em saúde. J. nurs. health. 2019;9(1):e199101

\section{RESUMO}

Objetivo: analisar a produção científica sobre as ações de saúde mental desenvolvidas no âmbito da Estratégia Saúde da Família. Metodologia: revisão integrativa de literatura realizada na Biblioteca Virtual em Saúde, PubMed e Web of Science, que após aplicação dos critérios, selecionaram-se 14 artigos científicos. Resultados: as ações de saúde mental desenvolvidas na Saúde da Família são de matriciamento, o Programa Intervenção Precoce, a Terapia Comunitária Integrativa, os grupos terapêuticos e a visita domiciliar. Também foi identificado o desenvolvimento de práticas focadas na doença com o privilégio de consultas ambulatoriais e o uso excessivo de psicofármacos. Há necessidade de investimentos na formação do profissional da saúde, além do fortalecimento da rede extra-hospitalar que sirva de retaguarda para a Saúde da Família. Conclusão: a literatura aponta que o cuidado em saúde mental na Saúde da Família é tímido e ainda muito focado no modelo biomédico. Descritores: Saúde mental; Estratégia saúde da família; Assistência integral à saúde.

\section{ABSTRACT}

Objective: to analyze scientific production on mental health actions developed in the Family Health Strategy. Methodology: integrative review of the literature in the Health Virtual Library, PubMed and Web of Science, that after applying the criteria, 14 scientific articles were selected. Results: the mental health actions developed in the Family Health are matricial practice strategies, the earlyintervention programmes, Integrative Communion, therapeutic groups and household visit. The

1 Enfermeira. Mestre em Enfermagem. Universidade Federal do Rio Grande do Sul (UFRGS). E-mail: adrianeeslabao@hotmail.com http://orcid.org/0000-0003-1262-6521

2 Enfermeira. Mestre em Enfermagem. Universidade Federal do Rio Grande do Sul (UFRGS). E-mail: elitiele_ortiz@hotmail.com http://orcid.org/0000-0002-2537-6069

3 Enfermeira. Doutora em Enfermagem. Faculdades Integradas de Taquara (FACCAT). E-mail: vilmacfsantos@gmail.com http://orcid.org/0000-0003-1075-1871

4 Enfermeira. Mestre em Enfermagem. Universidade Federal do Rio Grande do Sul (UFRGS). E-mail: betty.83@hotmail.com http://orcid.org/0000-0002-3017-5560

5 Enfermeira. Mestre em Enfermagem. Universidade Federal do Rio Grande do Sul (UFRGS). E-mail: ritamello42@gmail.com http://orcid.org/0000-0002-0032-9055

6 Enfermeiro. Doutor em Enfermagem. Universidade Federal do Rio Grande do Sul (UFRGS). E-mail: jaco_schneider@uol.com.br http://orcid.org/0000-0002-0151-3612 


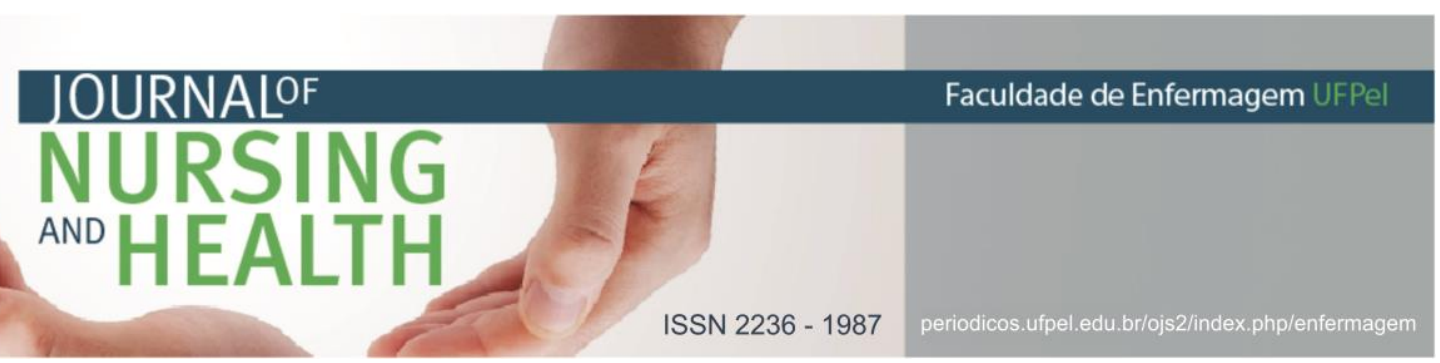

development of disease-focused practices was also identified with the privilege of ambulatory consultations and the excessive use of psychiatric drugs. There is a permanent necessity of investments in the training of health and education professionals, besides the strengthening of the extra hospital network, serving as a backup for the family health. Conclusion: the literature points out that mental health care in the family health is timid and still too much focused in the biomedical model.

Descriptors: Mental Health; Family Health Strategy; Comprehensive Health Care Integral Health Assistance.

\section{RESUMEN}

Objetivo: analizar la producción científica sobre las acciones de salud mental en el ámbito de la Estrategia Salud de la Familia. Metodologia: revisión integrativa realizada en la Biblioteca Virtual de Salud, PubMed y Web of Science, y después de la aplicación de los criterios se seleccionaron 14 artículos. Resultados: las acciones son de matriciamento, Programa Intervención Precoz, Terapia Comunitaria Integrativa, grupos terapéuticos y la visita domiciliar. También se identificaron prácticas enfocadas en la enfermedad con el privilegio de consultas ambulatorias y el uso excesivo de psicofármacos. Hay una necesidad de inversiones en la formación profesional de la salud y de la educación permanente, además del fortalecimiento de la red extra hospitalaria que puede servir de retaguardia para la salud de la familia. Conclusión: la literatura demuestra que el cuidado en salud mental en la salud de la familia es tímido y todavía muy enfocado en el modelo biomédico.

Descriptores: Salud mental; Estrategia de Salud Familiar; Atención Integral de Salud.

\section{INTRODUÇÃO}

A Reforma Psiquiátrica, acompanhando a Reforma Sanitária, tem lutado pela alteração de conceitos e práticas na forma como se oferece atenção às pessoas em sofrimento psíquico pelas políticas públicas. Assim visa à universalidade da assistência e a ampliação do acesso aos serviços de saúde por meio de ações a serem desenvolvidas pela atenção básica e a corresponsabilização da rede de serviços por esta demanda. ${ }^{1-2}$

A Atenção Básica em Saúde, "capilarizada" e concretizada por meio das Equipes de Saúde da Família, da Estratégia de Saúde da Família (ESF), tem recebido incumbências de grande monta, como a desafiadora responsabilidade de atender as demandas em Saúde Mental. Isto exige mais do que mudar elementos conceituais do plano teórico, mas também introduzir novas propostas metodológicas de ação que exigem transformações práticas e concretas que emanam do cotidiano. ${ }^{1}$

No contexto brasileiro a atenção básica assume um lugar estratégico na constituição da rede de cuidados e é considerada a principal porta de entrada no sistema de saúde, este nível de atenção desempenha um papel importante junto ao campo da saúde mental dada a proximidade territorial as famílias. Esta proximidade proporciona a identificação e a assistência das pessoas em sofrimento psíquico e as famílias, assumindo a atenção psicossocial como um modo de cuidar. $^{2-3}$

Este nível de atenção tem potencial para atuar em duas grandes frentes de ações em saúde mental, a primeira é pensada pela perspectiva de detectar as queixas relativas ao sofrimento psíquico e promover escuta 


\section{JOURNALOF \\ NURSING \\ AND HEALTH}

ISSN 2236 - 1987

qualificação das atividades e na busca pelos direitos de todos envolvidos. ${ }^{8-9}$

Assim, com o desejo de produzir um material que discuta e problematize as maneiras de como se dá a articulação e o diálogo entre o campo da Saúde Mental e a Atenção Básica em Saúde, este estudo teve por objetivo analisar a produção científica sobre as ações de saúde mental desenvolvidas no âmbito da Estratégia Saúde da Família (ESF).

\section{MATERIAL E MÉTODOS}

Este estudo trata-se de uma revisão integrativa de literatura, realizada a partir da disciplina de Fundamentos e Práticas de Enfermagem e Saúde, do Programa de Pós-Graduação em Enfermagem da Universidade Federal do Rio Grande do Sul.

A revisão integrativa é um método criterioso utilizado para fornecer os melhores conhecimentos produzidos sobre um dado problema e tem como finalidade a sistematização ordenada e abrangente dos resultados, constituindo assim um corpo de conhecimento. ${ }^{10}$

Para operacionalizar esta revisão integrativa foram realizadas seis fases: $1^{\circ}$ elaboração da pergunta norteadora; $2^{\circ}$ busca ou amostragem na literatura; $3^{\circ}$ coleta de dados; $4^{\circ}$ análise crítica dos estudos incluídos; $5^{\circ}$ discussão dos resultados e $6^{\circ}$ apresentação da revisão integrativa. ${ }^{11}$

A pergunta norteadora do estudo foi: "quais ações de saúde mental são desenvolvidas no contexto da Estratégia Saúde da Família?" elaborada na primeira fase. $\mathrm{Na}$ 
segunda fase, foram realizadas a captura das produções nas seguintes bases de dados: Biblioteca Virtual em Saúde (BVS), National Library of Medicine (PubMed) e Web of Science. $\mathrm{Na}$ BVS e Web of Science foram utilizando os Descritores em Ciências da Saúde (DeCS): "Saúde Mental" "Estratégia Saúde da Família" "Assistência Integral à Saúde", com o booleano AND. E na base de dados Pubmed foram utilizados os Mesh termos: "Mental Health" "Family Health" "Comprehensive Health Care", com o booleano And.

Estabeleceu-se como critérios de inclusão no estudo: artigos científicos disponíveis online na íntegra por meio eletrônico, publicados entre janeiro de 2010 a dezembro de 2015, o período foi definido com o intuito de agrupar pesquisas recentes, nos idiomas de português, inglês e espanhol. A investigação cientifica da pesquisa foi realizada no período de março a maio de 2016.

$\mathrm{Na}$ terceira fase de coleta de dados foi realizada a leitura dos títulos e resumos de todos os documentos encontrados utilizando-se os seguintes critérios de exclusão: artigos repetidos; que não se enquadrasse na temática e/ou na questão de pesquisa; teses, dissertação e monografia. Deste modo, foram encontrados 99 documentos relacionados à temática do estudo e destes 71 foram excluídos por não se enquadrar à temática e/ou não corresponderem à questão de pesquisa, dois por serem trabalhos de conclusão de curso e/ou de pósgraduação e 12 por estarem repetidos. Assim, resultou-se em 14 artigos científicos que foram analisados e utilizados neste estudo (Quadro 1).

Quadro 1: Critérios para exclusão dos artigos.

\begin{tabular}{|l|r|r|r|r|}
\hline \multicolumn{1}{|c|}{ Critérios } & \multicolumn{1}{|c|}{ BVS } & PubMed & $\begin{array}{r}\text { Web of } \\
\text { Science }\end{array}$ & Total \\
\hline Total localizado & 38 & 20 & 41 & 99 \\
\hline Artigos repetidos & 3 & 3 & 66 & 12 \\
\hline $\begin{array}{l}\text { Não se enquadra à temática / não corresponde à } \\
\text { questão de pesquisa }\end{array}$ & 21 & 17 & 33 & 71 \\
\hline Excluído por ser tese, dissertação e monografia. & 2 & 0 & 0 & 2 \\
\hline Total selecionado & 12 & 0 & 2 & 14 \\
\hline
\end{tabular}

$\mathrm{Na}$ quarta fase de análise crítica dos 14 estudos incluídos foi realizada a leitura na integral de todos os artigos científicos, selecionando as seguintes informações: base de dados, objetivo do estudo, tipo de estudo e principais resultados e conclusões de acordo com os objetivos do estudo. Incluíram-se os artigos de avaliação, análise, e discussões envolvendo as ações de saúde mental na Estratégia Saúde Família. Os dados foram descritos em planilhas do software Microsoft Office Excel ${ }^{\circledR} 2007$.

$\mathrm{Na}$ quinta e na sexta fases foi realizada a análise dos resultados 


\section{JOURNALOF \\ NURSING \\ AND HEALTH}

ISSN 2236 - 1987

revisão de literatura. Quanto aos estados em que foram desenvolvidas as pesquisas: seis estudos na região nordeste; três na região sudeste, dois na região Sul, um no Distrito Federal e dois não há identificação por se tratar de revisão integrativa, todos os estudos foram realizados no Brasil.

Em relação aos participantes do estudo: cinco pesquisas foram realizadas com profissionais de saúde, dois estudos envolveram profissionais de saúde, usuários e familiares, dois estudos envolveram profissionais $\mathrm{e}$ usuários, um estudo com profissionais e estudantes de medicina e enfermagem, um estudo envolvendo só usuários, uma pesquisa documental e dois estudos eram revisão integrativa.

Referente a autoria dos artigos, identificou-se quatro artigos com composição multidisciplinar, entre eles um artigo de Fonoaudiólogos com Enfermeiros; um artigo de Assistente social com Enfermeiros; um artigo de Médicos, Enfermeiros, Odontólogos e Psicólogos; um artigo de Médicos e Psicólogos. Seis artigos apresentavam autoria somente de Enfermeiros; um de médicos; um de psicólogos. Dois artigos não apresentavam informações a respeito da formação dos autores, somente as instituições as quais eram vinculados.

De acordo com o ano de publicação, foram encontradas cinco (35,7\%) publicações no ano de 2012; três publicações em 2011 e 2013; duas publicações em 2014; e uma em 2015. 
Quadro 2: Artigos que compõem a amostra da revisão integrativa localizados na BVS e Web of Science, compreendendo o período de janeiro de 2010 a dezembro de 2015.

\begin{tabular}{|c|c|c|c|}
\hline Título do artigo & $\begin{array}{l}\text { Metodologia } \\
\text { de pesquisa }\end{array}$ & Periódico & Ano \\
\hline $\begin{array}{l}\text { A práxis da saúde mental no âmbito da } \\
\text { estratégia saúde da família: contribuições } \\
\text { para a construção de um cuidado } \\
\text { integrado }\end{array}$ & $\begin{array}{l}\text { Qualitativa e } \\
\text { Quantitativa }\end{array}$ & $\begin{array}{l}\text { Physis, Revista de Saúde } \\
\text { Coletiva }\end{array}$ & 2011 \\
\hline $\begin{array}{l}\text { Apoio matricial como dispositivo do cuidado } \\
\text { em saúde mental na atenção primária: } \\
\text { olhares múltiplos e dispositivos para } \\
\text { resolubilidade }^{13}\end{array}$ & Qualitativa & $\begin{array}{l}\text { Revista Ciência e Saúde } \\
\text { Coletiva }\end{array}$ & 2012 \\
\hline $\begin{array}{l}\text { Apoio matricial em saúde mental na } \\
\text { atenção primária à saúde: uma análise da } \\
\text { produção científica e documental }{ }^{14}\end{array}$ & $\begin{array}{l}\text { Revisão de } \\
\text { Literatura }\end{array}$ & $\begin{array}{l}\text { Interface: Comunicação, } \\
\text { Saúde e Educação }\end{array}$ & 2013 \\
\hline $\begin{array}{l}\text { Atendimento ao indivíduo com transtorno } \\
\text { mental: perspectiva de uma equipe da } \\
\text { estratégia de saúde da família }{ }^{15}\end{array}$ & Qualitativa & $\begin{array}{l}\text { Revista Baiana de } \\
\text { Enfermagem }\end{array}$ & 2014 \\
\hline $\begin{array}{l}\text { Avaliação da satisfação dos usuários com o } \\
\text { cuidado da saúde mental na estratégia } \\
\text { saúde da família尚 }\end{array}$ & Qualitativa & $\begin{array}{l}\text { Revista da Escola de } \\
\text { Enfermagem da USP }\end{array}$ & 2011 \\
\hline $\begin{array}{l}\text { Avaliação de estratégias inovadoras na } \\
\text { organização da Atenção Primária à Saúde }{ }^{17}\end{array}$ & Qualitativa & Revista Saúde Pública & 2012 \\
\hline $\begin{array}{l}\text { Concepções dos profissionais da estratégia } \\
\text { saúde da família sobre intervenção precoce } \\
\text { em saúde mental }{ }^{18}\end{array}$ & Qualitativa & $\begin{array}{l}\text { Revista Ciência e Saúde } \\
\text { Coletiva }\end{array}$ & 2013 \\
\hline $\begin{array}{l}\text { Ferramenta matricial na produção do } \\
\text { cuidado integral na estratégia saúde da } \\
\text { família }^{19}\end{array}$ & Qualitativa & $\begin{array}{l}\text { Acta Paulista de } \\
\text { Enfermagem }\end{array}$ & 2012 \\
\hline $\begin{array}{l}\text { Formação de terapeutas comunitários na } \\
\text { Paraíba: impacto na estratégia saúde da } \\
\text { família }^{20}\end{array}$ & $\begin{array}{l}\text { Quantitativa e } \\
\text { Qualitativa }\end{array}$ & $\begin{array}{l}\text { Revista Eletrônica de } \\
\text { Enfermagem }\end{array}$ & 2012 \\
\hline $\begin{array}{l}\text { Percepção dos profissionais de saúde e } \\
\text { comunitários em relação à terapia } \\
\text { comunitária na estratégia saúde da } \\
\text { família }^{21}\end{array}$ & Qualitativa & Revista APS & 2012 \\
\hline $\begin{array}{l}\text { Práticas em saúde mental na estratégia } \\
\text { saúde da família: um estudo exploratório } 22\end{array}$ & Qualitativa & $\begin{array}{l}\text { Revista Pesquisa Cuidado } \\
\text { é Fundamental }\end{array}$ & 2015 \\
\hline $\begin{array}{l}\text { Psiquiatria no século XXI: transformações a } \\
\text { partir da integração com a atenção } \\
\text { primária pelo matriciamento }{ }^{23}\end{array}$ & Qualitativa & $\begin{array}{l}\text { Physis Revista de Saúde } \\
\text { Coletiva }\end{array}$ & 2014 \\
\hline $\begin{array}{l}\text { Saúde mental na atenção básica: } \\
\text { possibilidades para uma prática voltada } \\
\text { para a ampliação e integralidade da saúde } \\
\text { mental }{ }^{24}\end{array}$ & Qualitativa & $\begin{array}{l}\text { Revista Saúde e } \\
\text { Sociedade }\end{array}$ & 2013 \\
\hline $\begin{array}{l}\text { Visita domiciliar na atenção à saúde } \\
\text { mental }^{25}\end{array}$ & $\begin{array}{l}\text { Revisão de } \\
\text { Literatura }\end{array}$ & Ciencia y Enfermería XVI & 2011 \\
\hline
\end{tabular}




\section{DISCUSSÃO}

O método de análise do material possibilitou interpretar e agrupar os dados semelhantes. Desse agrupamento emergiu três unidades de análise, que serão apresentas a seguir.

Ações de saúde mental no contexto da Estratégia Saúde Família

As ações de saúde mental desenvolvidas no contexto da Estratégia Saúde da Família que emergiram na revisão integrativa foram ações de matriciamento ${ }^{12-17}$, o Programa Intervenção Precoce ${ }^{18}$, a Terapia Comunitária Integrativa ${ }^{20-21}$, os grupos terapêuticos e a visita domiciliar. ${ }^{24-25}$

O apoio matricial aparece como ferramenta de trabalho que favorece a interação entre equipes da atenção básica e equipes especializadas. Essa estratégia visa contribuir no cuidado de pessoas em sofrimento psíquico, no acesso, no fluxo, na diversificação e ampliação dos espaços de cuidado no território. ${ }^{13,19}$

Trata-se de um novo modo de produzir cuidado em um processo de construção compartilhada com duas ou mais equipes de saúde, construindo um modo de intervenção pedagógicoterapêutica, na qual as equipes da atenção básica são instrumentalizadas para lidar com as demandas em saúde mental. Desde 2003, o Ministério da Saúde, prioriza o apoio matricial como a forma de organização das ações de saúde mental junto à atenção básica. ${ }^{12}$

Nesse sentido, os municípios brasileiros têm incorporado o apoio
ISSN $2236-1987$

matricial como uma metodologia de trabalho com a intenção de partilhar a responsabilidade pelo cuidado em saúde mental, incentivando outros setores da saúde para estas demandas e superando a lógica dos encaminhamentos aos especialistas, apontando espaços e possibilidades para o fortalecimento da saúde mental dos indivíduos que emanam dos próprios territórios, buscando a prevenção do adoecimento e a promoção da saúde. ${ }^{2,26}$

Diante do potencial de integração dos campos sociais e a proximidade com os espaços de vida, as práticas de saúde mental na atenção básica contribuem para a realização de ações voltadas prioritariamente para a promoção da cidadania e a construção da autonomia da população. Elementos importantes para a viabilização da Reforma Psiquiátrica. ${ }^{27}$

0 fato do matriciamento ter aparecido nessa revisão é valioso e traz à tona a necessária discussão de sua importância como estratégia de fortalecimento das ações de saúde mental na atenção básica. No entanto, ainda é preciso avanços na implantação dessa ferramenta, uma vez que na prática o encontro entre as equipes precisa ser regular, ter a participação ativa dos profissionais, uma proposição de assistência conjunta entre os profissionais do Centro de Atenção Psicossocial (CAPS) e da Unidade Básica de Saúde (UBS) e do Núcleo de Apoio a Saúde da Família (NASF). ${ }^{17,28}$

Dessa forma, a correta implantação do apoio matricial possibilita uma ampliação no cuidado em saúde mental, tendo a Estratégia 
Saúde da Família como protagonista desta atenção. Neste sentido, é preciso uma integração entre as equipes de ESF e as equipes do CAPS e NASF para garantir respostas resolutivas de cuidado da população em sofrimento psíquico. ${ }^{28}$

$\mathrm{Na}$ presente revisão, outra importante ação em saúde mental que aparece na atenção básica é o cuidado ao público infantil. A qualificação do cuidado em saúde mental a criança é apontada como uma necessidade das equipes tanto no âmbito da prevenção de complicações do desenvolvimento infantil, bem como para melhor promover a inclusão da criança na família e na sociedade. ${ }^{18}$

É importante que crianças e adolescentes tenham acesso a espaços acolhedores juntamente com seus familiares e que os profissionais desenvolvam ações que busquem o cuidado com ênfase no desenvolvimento individual e social de cada criança. ${ }^{29}$

A identificação precoce de alterações, tratamento e reabilitação das crianças com algum transtorno evita a ocorrência de encaminhamentos desnecessários para a atenção especializada e quando necessários, os torna mais qualificados contribuindo para a agilidade do fluxo referência- contrareferência. ${ }^{30}$

Levando em consideração as especificidades desse cuidado, vem sendo desenvolvido o Programa de Intervenção Precoce no município de Campina Grande-PB. O Programa Municipal Intervenção Precoce (PMIP) se caracteriza por dar suporte às famílias e às crianças de zero a seis anos que têm ou correm o risco de desenvolver algum transtorno e/ou atraso no desenvolvimento devido às condições ambientais, sociais e/ou biológicas. Essa experiência é uma estratégia inovadora local gestada em experiências que fortalecem a manutenção da rede de saúde mental e sua interação com serviços de atenção básica. ${ }^{18}$

No PMIP, as práticas profissionais também devem estar direcionadas para a escuta atenta e problematizadora dos conteúdos do imaginário materno e também no reconhecimento de recursos junto à família para a construção de habilidades e competências para lidar com as dificuldades implícitas no processo. ${ }^{30}$

Uma das principais dificuldades enfrentadas pelos profissionais da ESF na Intervenção Precoce é a insegurança em algumas ações, entre elas o diagnóstico das psicopatologias, a avaliação sobre a necessidade de encaminhamentos para o serviço especializado e o conhecimento sobre a saúde mental. ${ }^{18}$

Tais desafios apontam a necessidade de investir na qualificação dos profissionais da ESF para o desenvolvimento de ações em saúde mental, melhorar integração com a escola, e com a sociedade civil e com os serviços de saúde mental especializados. Além dessas estratégias, é fundamental fortalecer a parceria com a família, incorporando no cuidado elementos ambientais, culturais e sociais com vistas a aproximar a equipe, a família e o território. ${ }^{18,31}$ 


\section{JOURNALOF \\ NURSING \\ ANO HEALTH}

A experiência do Programa de Intervenção Precoce, apesar de ser recente e apresentar muitos desafios, demonstra o papel da atenção básica enquanto estratégia no cuidado em saúde mental, articulando em suas ações a perspectiva da prevenção em saúde mental e responsabilização sobre os casos de saúde mental infantil no território.

Acredita-se que o êxito desse tipo de experiência se dá em função do apoio de uma gestão municipal comprometida com os princípios do SUS e da Reforma Psiquiátrica. No entanto, a manutenção dessas ações exige um envolvimento da rede especializada a fim de dar suporte para os profissionais da ESF, buscando identificar as crianças que não chegam até o serviço.

É preciso construir coletivamente uma rede de cuidados que permita aos profissionais da atenção primária identificar as alterações de desenvolvimento e presença de transtornos, contando com o apoio de profissionais especializados, se necessário. E também identificar as possibilidades que estão envolvidas nos diversos cenários em que a criança transita, seja a escola, a praça, as quadras de esportes, projetos sociais entre outros. $32-33$

Outra estratégia de Atenção Psicossocial desenvolvida no Município de Jaboatão dos Guararapes/PE e no contexto da Atenção Básica é a Terapia Comunitária Integrativa ( $\mathrm{TCl})$, considerada um tipo de abordagem para o cuidado em grupo, com a finalidade de promover a saúde e saúde mental na atenção primária. É uma prática que valoriza a essência
ISSN $2236-1987$

humana, promove práticas de escuta, compreensão e desenvolve laços de afeto entre a própria comunidade e os profissionais ${ }^{21}$, isso é o que diferencia a $\mathrm{TCl}$ de outros grupos terapêuticos.

Foram identificados muitos benefícios a partir do desenvolvimento dessa prática. Para os profissionais, sujeitos da pesquisa e que participaram de pelo menos um encontro ou roda de $\mathrm{TCl}$. A TCl ajuda a compreender melhor as pessoas, a comunidade e seus problemas, contribuindo para melhorar a relação e o vínculo entre profissionais e a comunidade. ${ }^{21}$

Para a comunidade a $\mathrm{TCl}$ promoveu um melhor controle sobre suas emoções, o exercício de repensar melhor sobre os problemas e resolvelos, conhecer melhor a si mesmo e as suas capacidades individuais, tendo mais confiança em suas ações e atitudes. Também promoveu a socialização, o exercício da escuta e da fala no grupo, a união e a construção de laços de convivência, afeto e empatia. ${ }^{21}$

Para o desenvolvimento da $\mathrm{TCl}$ o profissional precisa de formação e apoio. 0 curso de formação em Terapia Comunitária Integrativa trabalha o autoconhecimento, a autoestima, a segurança, paciência e outros aspectos para que o terapeuta se torne capaz de coordenar uma roda de terapia conseguindo mediar as relações, as falas e as emoções que emergirem. ${ }^{20} 0$ artigo cita de modo geral os técnicos, profissionais da área de saúde e educação, principalmente profissionais das Equipes de Saúde da Família, como possíveis terapeutas aptos a realizar a $\mathrm{TCl}$. 


\section{JOURNALOF \\ NURSING \\ AND HEALTH}

A $\mathrm{TCl}$ é um instrumento de rico valor grupal, acolhedor e expressivo que contribui para a reconstrução de identidades, de resgate de autoestima, valorização de saberes, falas e escuta próprias, considerado uma prática muito eficiente e eficaz para a promoção e prevenção da saúde mental. ${ }^{34-35}$

A $\mathrm{TCl}$ por meio do fortalecimento de vínculos positivos promove redes solidárias de apoio e otimiza as potencialidades $e$ os recursos da comunidade, o que é de suma importância quando nos deparamos com a insuficiência ou fragilidade de serviços existentes para atender as demandas. ${ }^{36}$

Dessa forma, percebe-se que a $\mathrm{TCl}$ pode proporcionar um importante espaço para intervenções em saúde mental na atenção básica. Apostar em espaços que possibilite a construção de uma rede de apoio com conexões entre a própria comunidade e os profissionais é disparador para o estabelecimento de uma outra relação de cuidado, na qual o objeto de intervenção passa a ser a pessoa, e não a doença.

No que se refere ao contexto da prática da Atenção Primária à Saúde (APS), há outras ações que também podem ser fomentadas e melhor trabalhadas para avançar na qualificação do cuidado em saúde mental, entre elas destaca-se 0 desenvolvimento de grupos, não apenas a $\mathrm{TCl}$ que também é um grupo, e visitas domiciliares.

Os grupos apresentam grande potencial para serem utilizados como ferramenta terapêutica e como estratégia de promoção de saúde. ${ }^{37}$
ISSN 2236 - 1987

Podem ser desenvolvidos tanto na proposta de um grupo terapêutico como de um grupo operativo, pois dessas duas propostas emergem assuntos ligados à subjetividade dos participantes e que podem promover dispositivos pessoais para trabalharem com eventos adversos, traumáticos e frageis. ${ }^{24}$

As visitas domiciliares possibilitam conhecer melhor a subjetividade que envolve o sistema familiar, compreendendo não apenas o olhar para o sujeito, mas para toda a sua família e o contexto, incluindo-os nesse processo de cuidado e planejamento de estratégias terapêuticas. ${ }^{24,38}$

Dessa forma, o domicílio passa a funcionar como espaço terapêutico, e o atendimento humanizado torna-se cada vez mais viável, criando um vínculo entre a equipe e a família e maiores possibilidades de intervenção, inclusive para que sejam trabalhados, o preconceito, a exclusão da pessoa em sofrimento psíquico que muitas vezes ocorre no interior do núcleo familiar. ${ }^{25}$

Os grupos e as visitas domiciliares são potentes instrumentos de trabalho em saúde mental na atenção básica e se configuram em verdadeiros métodos integrais de atenção, mas é preciso um olhar ampliado em relação ao modo de fazer e realizar este cuidado. ${ }^{24}$ Assim, é preciso um deslocamento dos processos de trabalho em saúde, uma reorganização dos serviços e dos modos de produção do cuidado, é preciso pôr em prática um modelo ampliado de cuidado no qual o usuário é o centro da atenção. ${ }^{39}$ 


\section{JOURNAIOF \\ NURSING \\ ANO HEALTH}

Diante dessas considerações, o matriciamento desvela-se como uma das estratégias mais potentes, que através do incentivo político, vem sendo concretizada em muitos municípios demonstrando sua inovação pela possibilidade de diálogo, apoio, e aprendizado entre as equipes. Também aparecem estratégias locais como intervenção precoce e a terapia comunitária integrativa que precisam de melhor divulgação e interesse da gestão e dos profissionais para ser desenvolvida em outros locais. No entanto, percebe-se que as ações de saúde mental na atenção básica ainda acontecem de forma tímida através da visita domiciliar e desenvolvimento de grupos.

\section{Ações biomédicas no contexto da} ESF e seus desafios para o campo da saúde mental

Nesta revisão integrativa, as práticas biomédicas aparecem de forma considerável na atenção básica e são representadas por práticas de atenção focadas na doença e nos sintomas $^{12-13,24,40-41}$, o que gera um desafio no cuidado na perspectiva da integralidade das ações nesse âmbito da atenção.

Os artigos analisados apresentam ações de cuidado que privilegiam a prescrição de psicotrópicos e o predomínio de procedimentos com enfoque no indivíduo e no agravo, com encaminhamentos para internações em detrimento de ações de cuidado que podem ser desenvolvidas na ESF. 1213,24,40-41 Estas dificuldades podem estar relacionadas com o despreparo dos profissionais para atuar, identificar e cuidar precocemente das pessoas com
ISSN $2236-1987$

transtornos mentais nas ESF, o que leva aos constantes encaminhamentos dos usuários para os serviços especializados. ${ }^{42-43}$

Em um estudo, realizado com usuários, familiares e profissionais de CAPS e ESF com o objetivo de analisar a articulação entre as equipes destes dois serviços; identificou-se a prática da consulta médica como única forma de resolução dos problemas de saúde. $\mathrm{Na}$ ESF as práticas de cuidado estavam voltadas para as medidas prescritivas com procedimentos de controle e intervenções programáticas do processo saúde e doença, acarretando resistência para o cuidado de pessoas em sofrimento psíquico. ${ }^{13}$

Nos registros de cuidado em saúde mental realizados na ESF a dispensação de medicamentos psicotrópicos e o seu controle é o principal registro, acrescentado das anotações de referência e contrarreferência para os serviços especializados. Deste modo, os registros revelam pouco sobre a real situação vivenciada pela população em sofrimento psíquico. ${ }^{13}$

Ainda, os autores destacam como dispositivo terapêutico de efetivação no cuidado a corresponsabilização entre os profissionais e usuários incorporando a família, o território e as atividades intersetoriais no contexto socio-comunitário.

corresponsabilização possibilita o compartilhamento de problemas vivenciados e a pactuação integrada para a resolução. Assim, assumir responsabilidades que almejam a qualidade de vida, individual ou coletiva, trazem resultados mais resolutivos. ${ }^{13}$ 
$\mathrm{Na}$ regional de Brazlândia, Distrito Federal, foi realizado uma pesquisa com 26 profissionais de equipes de ESF, com o objetivo de analisar as práticas destes trabalhadores em relação às ações desenvolvidas no cuidado à Saúde Mental. Nesta pesquisa, identificou-se práticas de atenção focadas em consultas ambulatoriais médicas e encaminhamentos para internações, destacando baixa capacidade de resolução do sofrimento psíquico no âmbito da saúde da família. Além disso, evidenciou-se a ausência de uma rede de apoio estruturada, a desarticulação dos serviços existente acarretando dificuldade no acesso ao cuidado em saúde mental. ${ }^{12}$

Porém, mesmo a equipe focando o cuidado especializado na figura do médico, percebeu-se que os profissionais buscam atividades diversificadas em outros espaços sociais para inserir as pessoas com sofrimento psíquico como forma de cuidado. ${ }^{12}$

Em outro estudo realizado com os profissionais de uma equipe de saúde da família identificou-se uma concepção de saúde mental como doença/transtorno mental, havendo poucos modos de cuidado com caráter de prevenção e promoção à saúde mental. A pesquisa evidenciou que os usuários estão condicionados ao uso continuo de psicofármacos como forma de aliviar suas tristezas e angustias da vida, além disso, existe um olhar enraizado no discurso dos trabalhadores da saúde como a pessoa em sofrimento psíquico sendo 0 "diferente", o não normal. ${ }^{24}$
$\mathrm{Na}$ visão destes profissionais a existência de um diagnóstico em saúde mental do usuário implicaria no encaminhamento do mesmo para o CAPS, o serviço que possui uma equipe especializada para realizar o cuidado. Deste modo, percebe-se que a equipe não utiliza o acolhimento como forma de acolher a subjetividade e as dores que permeiam o usuário, mas como uma prática para atendimento da demanda e do que está explícito e emergencial. ${ }^{24}$

Em outra pesquisa ${ }^{22}$ as ações de saúde mental seguiam também uma tendência biologicista com 0 persistente tratamento farmacológico e o foco no adoecimento traduzido em sinais e sintomas e com os encaminhamentos para serviços especializados, provocando barreiras ao acesso de usuários que realmente precisam dos serviços como o CAPS devido a sua superlotação. O estudo evidenciou, ainda, um desconhecimento da equipe em relação ao potencial dos CAPS, de suas atribuições e de seu papel como regulador, bem como uma desarticulação entre as equipes de ESF e este serviço especializado.

Em outro estudo realizado com todos os profissionais de uma ESF que objetivou caracterizar o atendimento dos pacientes com demandas de saúde mental, bem como o conhecimento da equipe em relação a definição de transtorno mental e da reabilitação psicossocial. Evidenciou-se diferenças na conduta dos profissionais e o desconhecimento em relação a conduta dos colegas, principalmente a dos Agentes Comunitários de Saúde (ACS), a falta de atividades planejadas 
e especificas para este público e a dificuldades de comunicação entre os profissionais. ${ }^{40}$

Os profissionais definiram transtorno mental como "distúrbios" e "dificuldade mental" e alguns participantes não souberam responder, e em relação aos comportamentos e sintomas a maioria respondeu: agressividade, falta de noção de atitudes, agitação e falar sozinho. Em relação a reforma psiquiatra os participantes afirmam conhecê-la, mas observou-se um discurso desconecto da compreensão humana, o que dificulta a percepção da mudança de modelo de cuidado proposto. ${ }^{15}$

Estas limitações se fazem preocupantes, tendo em vista que os Transtornos Mentais Comuns (TMC) que são importantes problemas em saúde no Brasil e têm aumentado entre adolescentes escolares, e em idosos que com o avançar $\mathrm{da}$ idade apresentam com maior frequência morbidades psíquicas. Os TMC têm prevalência geral em torno de $55,8 \%$ na população idosa e com maior prevalência entre as mulheres. ${ }^{41-42}$

Para ultrapassar estas dificuldades é necessário superar o modelo clínico centrado na doença, tratamento e remissão de sintomas. A clínica em saúde mental desejada é aquela que busca se inserir nos modos de viver das pessoas e dos desejos que brotam dos projetos de vida, buscando outras formas de convívio em sociedade. ${ }^{44-45}$

Identifica-se muitos desafios para a consolidação do cuidado da saúde mental na atenção básica entre eles a necessidade de uma rede de apoio estruturada, melhor acesso aos serviços de saúde, pois há um número elevado de usuários à espera de consultas, o número de fichas insuficientes, a existência de filas, o tempo de espera para consulta e a insuficiência de médico ou de especialistas e o espaço físico inadequado. E a superação do uso excessivo de psicofármacos e a necessidade de espaços de educação permanente. ${ }^{12,16,22,24,46}$

A articulação e o diálogo são desafios das equipes de saúde da família, o não diálogo com as equipes de referência especializada leva ao não atendimento integral e transdisciplinar do cuidado. ${ }^{15,22,44,47-49}$ É preciso investir no diálogo, nas discussões em saúde mental na atenção primária, pois isso proporciona aos trabalhadores da ESF uma apropriação dos casos, promovendo a aproximação entre as equipes e a resolubilidade dos casos. ${ }^{16,48,50}$

Deste modo, é preciso ampliar o olhar em relação ao cuidado, investir em ações que promovam a escuta, o vínculo, o acolhimento, o autocuidado, a inserção social e participação da pessoa em sofrimento no seu próprio tratamento. Os desafios impostos pela dificuldade de acesso, da ausência de uma rede estruturada e da falta de comunicação não podem ser colocados como obstáculos para as ações em saúde mental, muito pode ser feito no cotidiano dos serviços de saúde, entretanto, é preciso unir forças para diminuir esses entraves do cuidado. 


\section{JOURNALOF \\ NURSING \\ aNOHEALTH}

Perspectivas para avanços da saúde mental na Atenção Básica

Para uma nova concepção e consequentemente uma nova prática de saúde mental na atenção Básica os artigos apontam a necessidade de reformulações no ensino na área da saúde $^{15,25,44}$, o investimento em educação permanente ${ }^{12,15,22-23,25} 0$ fortalecimento de uma clínica ampliada com parcerias entre a rede de saúde e intersetorial. ${ }^{12-13,24,44}$

A atenção básica é um novo campo de atuação da atenção em saúde mental e traz desafios importantes para a formação e o cuidado neste campo, neste sentido, o preparo profissional e a aproximação com as pessoas em sofrimento psíquico na comunidade, são fundamentais na qualidade assistencial. $23,25,51$

A qualificação das práticas em saúde mental é um tema relevante, tendo em vista que, mesmo depois de uma considerável trajetória da Reforma Psiquiátrica Brasileira, ainda há inadequação quanto ao atendimento aos grupos de familiares e a formação voltada à saúde mental, mesmo em serviços especializados. ${ }^{52}$

A fragilidade na formação profissional é apontada como um dos principais fatores que dificultam uma abordagem de cuidado integral do indivíduo e toda sua complexidade de saúde e psicossocial. Disso emergem outras dificuldades como a atenção em saúde mental ser pouco desenvolvida no contexto da Atenção Primária em Saúde. Um caminho para que isso fosse amenizado seria a qualificação dos profissionais no escopo dos recursos assistenciais, como o atendimento em
ISSN $2236-1987$

grupo, ações de prevenção de adoecimentos e de promoção da saúde. Assim, como a qualificação dos profissionais no que tange 0 gerenciamento das ações, a capacidade das equipes em estratificar riscos e registrar o acompanhamento e evolução de casos graves. ${ }^{53}$

Identifica-se que o modelo biomédico ainda é muito presente na formação do profissional da área da saúde de modo que as práticas são voltadas para a dimensão do cuidado com corpo físico e estratégias que buscam a cura. Dessa forma, a pessoa em sofrimento psíquico é vista apenas em suas questões orgânicas e a integração de recursos necessários para lidar com o sofrimento mental nas comunidades é pouco trabalhada na universidade. ${ }^{44}$

Quando não se conhece de maneira acurada os fatores que envolvem o sofrimento psíquico e o contexto político-social em que 0 próprio trabalho se insere, torna-se difícil visualizar e implementar estratégias para atender a esta demanda. Tal dissonância contribui para a manutenção de um modelo médico-centrado, que aparece na realidade de muitas ESF. ${ }^{15,25,44}$

Atualmente, com as novas Diretrizes Curriculares Nacionais, muitas universidades reformularam os currículos, buscando integrar os conteúdos de maneira a minimizar a fragmentação do ser humano. As metodologias ativas de ensino permitem ao estudante exercitar a reflexão, o senso crítico e a tomada de decisões, comprometendo-se com a implementação das políticas públicas 


\section{JOURNALOF \\ NURSING \\ M०HEALTH}

ISSN 2236 - 198

de saúde e necessidade de saúde da população. ${ }^{15}$

Desses esforços emergem experiências que apontam para a inserção gradual de ações em saúde mental, são estratégias de cuidado incorporadas aos territórios, como as visitas domiciliárias, que ampliam a clínica neste campo. ${ }^{25,54}$ Nesse sentido, a formação acadêmica precisa ser alvo de maiores discussões a fim de que o ensino promova a incorporação de trabalhadores mais preparados para atuar com a saúde mental no território. ${ }^{15,21,44}$

Outro fator apontado, que dificulta o desenvolvimento de ações relacionadas as demandas de saúde mental na atenção básica, é a falta de estratégias de educação permanente nas equipes da ESF. Com base na problematização do cotidiano da ESF, a educação permanente, poderia auxiliar no reconhecimento das necessidades de saúde, e também na identificação dos recursos para esse cuidado. ${ }^{12,15,22-23,25}$

O Matriciamento pode ser um dos espaços privilegiados para a educação permanente devido ao caráter coletivo de suas ações em um processo que reúne a problematização e a articulação em equipe capaz de construir um processo relacional e complexo de intercâmbio entre diferentes profissionais podendo melhorar a interação com a multifacetada realidade humana. ${ }^{23}$

A educação Permanente concebida nos moldes do apoio matricial contrapõe as capacitações que utilizam estratégias pedagógicas centradas no conteúdo, que não são contextualizadas ao mundo real do trabalho. ${ }^{22}$

Nesse sentido, a prática de educação permanente pelo apoio matricial, visa uma qualificação técnica e política em um processo de supervisão e apoio contínuo, buscando dessa forma superar a lógica do encaminhamento, a submissão e a centralidade no profissional médico, e principalmente avançar na organização da demanda no território de modo a efetivar politicamente uma rede de cuidados, considerando os diferentes momentos de saúde e do adoecimento psíquico. ${ }^{12,23}$

Além da priorização da relação estreita com os territórios, um importante passo para a ampliação do acesso e melhora da qualidade de atenção em saúde mental em todos os níveis e pontos de atenção no âmbito do SUS é fazer com que a Rede de Atenção Psicossocial (RAPS) se efetive, buscando a descentralização e regionalização. Os profissionais da saúde têm importante responsabilidade em conhecer os dispositivos disponíveis na RAPS e nos demais setores que podem contribuir com o cuidado em saúde. ${ }^{49,54}$

Avançar nas ações de saúde mental da APS também envolve que essas equipes estejam dispostas a assumir seu papel estruturante na rede de cuidado, organizando suas ações preventivas e de acompanhamento dos casos de saúde mental do seu território. Os princípios da Integralidade e do fazer para uma clínica ampliada presente na organização dessas equipes impulsionam a construção de outras 


\section{JOURNALOF \\ NURSING \\ ANO HEALTH}

ISSN 2236 - 1987

ações têm potencial de inovação no território da atenção básica somandose a outras estratégias que caminhem na direção de provocar mudanças na relação entre a equipe ESF e a pessoa em sofrimento psíquico, visando superar estigmas, preconceitos, melhorar a integração e inserção social dessas pessoas no território.

Em relação as ações biomédicas de saúde mental no contexto da Estratégia Saúde da Família e seus desafios foram identificadas: o cuidado biomédico com 0 forte uso dos psicofármacos como principal meio de cuidado, a dificuldade de acesso aos serviços, a falta de uma rede estruturada, falta de diálogo entre os profissionais. Há uma necessidade de investimentos na formação do profissional da saúde e na educação permanente, além do fortalecimento da rede extra hospitalar que sirva de retaguarda para a ESF. Nesse sentido, ressalta-se o importante desafio da consolidação do cuidado em saúde mental na atenção básica, visto que um número considerável de artigos apontou um cuidado focado no modelo biomédico.

Destaca-se a importância do cuidado em saúde mental na ESF, por essa ser responsável pela atenção de todos os transtornos mentais leves, por conhecer a realidade local de seu território de ação podendo planejar as atividades de saúde que promovam intervenções precoces de cuidado e ações de promoção em saúde mental. Além disso, observa-se que a saúde mental está presente em todas as fases da vida humana sendo importantíssimo o seu desenvolvimento, enquanto Terapia Comunitária Integrativa. Destaca-se que essas duas últimas 


\section{NURSING \\ AND HEALTH}

ISSN 2236 - 1987

https://aps.ufjf.emnuvens.com.br/ap s/article/view/2059/849

3 Campiotto LG, Yamaguchi MU. Reforma psiquiátrica no Brasil: estratégias adotadas. Revista uningá [Internet]. 2015[acesso em $2017 \mathrm{fev}$ 15];43:86-90. Disponível em: https: / / www.mastereditora.com.br/p eriodico/20150501_135433.pdf

4 Trajano MP, Bernardes SM, Zurba MC. $O$ cuidado em saúde mental: caminhos possíveis na rede de atenção psicossocial. Cadernos brasileiros de saúde mental [Internet]. 2018[acesso em 2017 jun 10];10(25):20-37. Disponível em:

http: / / incubadora.periodicos.ufsc.br/ index.php/cbsm/article/view/5001/5 202

5 Frosi RV, Tesser CD. Práticas assistenciais em saúde mental na atenção primária à saúde: análise a partir de experiências desenvolvidas em Florianópolis. Ciênc. Saúde Colet. [Internet]. 2015[acesso em 2017 abr 02];20(10):3151-61. Disponível em:

http://www.scielo.br/scielo.php?pid= S1413-

$81232015001003151 \&$ script=sci_abstra ct\&tlng=pt

6 Rocha CC, Barzaghi N. Dificuldades encontradas pelos psicólogos nos centros de atenção psicossocial (CAPSad): desafios da formação à atuação profissional. Revista uningá [Internet]. 2015 [acesso em 2017 maio 21];24(2):105-13. Disponível em:

http://revista.uninga.br/index.php/u ningareviews/article/view/1687/1297

7 Anjos Filho NC, Souza AMP. A percepção sobre o trabalho em equipe multiprofissional dos trabalhadores de 


\section{ISSN $2236-1987$}

um centro de atenção psicossocial em Salvador. Interface comum. saúde educ. [Internet]. 2017[acesso em 2017 jun 21];21(60):63-76. Disponível em:

http://www.scielo.br/pdf/icse/v21n6 0/1807-5762-icse-1807-

576220150428.pdf

8 Barros AC, Nascimento KC, Silva LKB, Silva JVS. A estratégia saúde da família no processo de matriciamento da saúde mental na atenção básica. Revista desafios [Internet]. 2018[acesso em 2017 nov 19];5(1):121 7. Disponível

em:

https: / / webcache.googleusercontent. com/search?q=cache:KPNafpvZOu8J:h ttps://sistemas.uft.edu.br/periodicos /index.php/desafios/article/download $/ 4767 / 12769 /+\& c d=1 \& h l=p t-$

BR\&ct $=c \operatorname{lnk} \& g l=b r$

9 Santos $A B$, Silva GG, Pereira MER, Brito RS. Saúde mental, humanização e direitos humanos. Cadernos brasileiros de saúde mental [Internet]. 2018[acesso em 2017 set 21];10(25):1 19. Disponível em:

http: / /incubadora.periodicos.ufsc.br/ index.php/cbsm/article/viewFile/499 $0 / 5201$

10 Ercole FF, Melo LS, Alcoforado CLGC. Revisão integrativa versus revisão sistemática. REME rev. min. enferm. [Internet]. 2014[acesso em 2017 set 02];18(1):9-12. Disponível em:

http://www.reme.org.br/artigo/detal hes/904

11 Souza MT, Silva MD, Carvalho R. Revisão integrativa: o que é e como fazer. Enstein (São Paulo). [Internet]. 2010[acesso em 2017 set 02];8(1):102 6. Disponível

em: http://www.scielo.br/pdf/eins/v8n1/ pt_1679-4508-eins-8-1-0102.pdf

12 Arce VAR, Sousa MF, Lima MG. A práxis da saúde mental no âmbito da estratégia saúde da família: contribuições para a construção de um cuidado integrado. Physis (Rio J.). [Internet]. 2011[acesso em 2016 mar 21];21(2):541-60. Disponível em: http://www.scielo.br/pdf/physis/v21 n2/a11v21n2.pdf

13 Pinto AGA, Jorge MSB, Vasconcelos MGF, Sampaio JJC, Lima GP, Bastos VC, et. al. Apoio matricial como dispositivo do cuidado em saúde mental na atenção primária: olhares múltiplos e dispositivos para resolubilidade. Ciênc. Saúde Colet. [Internet]. 2012[acesso em 2016 abr 21];17(3):653-60. Disponível em: http://www.scielo.br/pdf/csc/v17n3/ v17n3a11.pdf

14 Bonfim IG, Bastos ENE, Gois CWL, Tofoli LF. Apoio matricial em saúde mental na atenção primária à saúde: uma análise da produção científica e documental. Interface comum. saúde educ. [Internet]. 2013[acesso em 2016 mar 21];17(45):287-300. Disponível em:

http://www.scielo.br/pdf/icse/v17n4 5/aop1013.pdf

15 Mainarde DC, Matos PCN, Zanetti ACG, Reisdorfer E, Miguel TLB. Atendimento ao indivíduo com transtorno mental: perspectiva de uma equipe da estratégia de saúde da família. Rev. baiana enferm. [Internet]. 2014[acesso em 2016 maio 21];28(1):69-78. Disponível em:

https://portalseer.ufba.br/index.php /enfermagem/article/view/9265/870 9 
ISSN 2236 - 1987

16 Coimbra VCC, Kantorski LP, Oliveira MM, Pereira DB, Nunes CK, Eslabão AD. Avaliação da satisfação dos usuários com o cuidado da saúde mental na Estratégia saúde da família. Rev. Esc. Enferm. USP. [Internet]. 2011[acesso em 2016 mar 21];45(5):1150-6. Disponível em:

http://www.scielo.br/pdf/reeusp/v45 n5/v45n5a17.pdf

17 Onocko-Campos RT, Campos GWS, Ferrer AL, Corrêa CRS, Madureira PR, Gama CAP, et al. Avaliação de estratégias inovadoras na organização da atenção primária à saúde. Rev. saúde pública (Online). [Internet]. 2012[acesso em 2016 abr 21];46(1):43$50 . \quad$ Disponível em: http://www.scielo.br/pdf/rsp/v46n1/ 2502.pdf

18 Carvalho RN, Gondim ACS, Azevedo EB, Cavalcanti PB, Ferreira Filha MO, Queiroz D. Concepções dos profissionais da estratégia saúde da família sobre intervenção precoce em saúde mental. Ciênc. cuid. saúde. [Internet]. 2013[acesso em 2016 abr 21];12(1):10-8. Disponível em:

http://periodicos.uem.br/ojs/index.p $\mathrm{hp} /$ CiencCuidSaude/article/view/1390 $0 / p d f$

19 Jorge MSB, Pinto DM, Vasconcelos MGF, Pinto AGA, Souza RS, Caminha ECCR. Ferramenta matricial na produção do cuidado integral na estratégia saúde da família. Acta Paul. Enferm. (Online). [Internet]. 2012[acesso em 2016 abr 21];25(n.esp.2):26-32. Disponível em: http://www.scielo.br/pdf/ape/v25ns pe2/pt_05.pdf

20 Araruna MHM, Ferreira Filha MO, Dias MD, Braga LAV, Moraes MN, Rocha
IA. Formação de terapeutas comunitários na Paraíba: impacto na Estratégia saúde da família. Rev. eletrônica enferm. [Internet]. 2012[acesso em 2016 maio 21];14(1):33-41. Disponível em:

https://www.revistas.ufg.br/fen/arti cle/view/15679/15554

21 Cisneiros VGF, Oliveira MLS, Amaral GMC, Cunha DM, Silva MJF. Percepção dos profissionais de saúde e comunitários em relação à terapia comunitária na estratégia saúde da família. Rev. APS. [Internet]. 2012[acesso em 2016 abr 21];15(4):468-78. Disponível em: https://aps.ufjf.emnuvens.com.br/ap s/article/view/1558/679

22 Martins AKL, Souza AMA, Vieira NFC, Pinheiro PNC, Braga VAB. Práticas em saúde mental na estratégia saúde da família: um estudo exploratório. Rev. pesqui. cuid. fundam. (Online). [Internet]. 2015[acesso em 2016 maio 21];7(1):1905-14. Disponível em: http://www.seer.unirio.br/index.php /cuidadofundamental/article/view/34 63/pdf_1427

23 Fortes S, Menezes A, Athié $\mathrm{K}$, Chazan LF, Rocha H, Thiesen J, et al. Psiquiatria no século XXI: transformações a partir da integração com a atenção primária pelo matriciamento. Physis (Rio J.). [Internet]. 2014[acesso em 2016 mar 21];24(4):1079-102. Disponível em: http://www.scielo.br/pdf/physis/v24 n4/0103-7331-physis-24-04-01079.pdf

24 Moliner J, Lopes SMB. Saúde mental na atenção básica: possibilidades para uma prática voltada para a ampliação e integralidade da saúde mental. Saúde e sociedade [Internet]. 


\section{NURSING \\ ANO HEALTH}

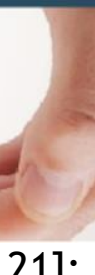

2013[acesso em 2016 maio 21]; 22(4):1072-83. Disponível em: http://www.scielo.br/pdf/sausoc/v22 n4/10.pdf

25 Silva CMC, Teixeira ER, Sabóia VM, Valente GSC. Visita domiciliar na atenção à saúde mental. Cienc. enferm. [Internet]. 2011[acesso em 2016 abr 21];17(3):125-36. Disponível em:

https://scielo.conicyt.cl/pdf/cienf/v1 7n3/art11.pdf

26 Santos EO, Coimbra VCC, Kantorski LP, Pinho LB, Andrade APM, Eslabão $A D$. Equipes de referência: contribuições para o trabalho em saúde mental. Pesqui. prát. psicossociais. [Internet]. 2018[acesso em 2017 nov 10];13(1):1-13. Disponível em: http://www.seer.ufsj.edu.br/index.p $\mathrm{hp} /$ revista_ppp/article/view/2817/18 40

27 Vasconcelos AMA, Silva DG. Concepção de cuidados em saúde mental na atenção primária à saúde. Revista interdisciplinar [Internet]. 2017 [acesso em 2017 maio 17];10(3):70-6. Disponível em: https://revistainterdisciplinar.uninova fapi.edu.br/index.php/revinter/articl e/view/1250

28 Wenceslau LD, Ortega F. Mental health within primary health care and global mental health: international perspectives and brazilian context. Interface comun. saúde educ. [Internet]. 2015[cited 2017 Mar 16];19(55):1121-32. Available from: http://www.scielo.br/pdf/icse/v19n5 5/en_1807-5762-icse-1807-

576220141152.pdf

29 Ministério da Saúde (BR). Atenção Psicossocial a crianças e adolescentes
ISSN 2236 - 1987

no SUS: tecendo redes para garantir direitos [Internet]. 2014[acesso em 2018 dez 10]. Disponível em: http://bvsms.saude.gov.br/bvs/public acoes/atencao_psicossocial_criancas_ adolescentes_sus.pdf

30 Ministério da Saúde (BR). A estimulação precoce na Atenção Básica: guia para abordagem do desenvolvimento neuropsicomotor pelas equipes de atenção básica, saúde da família e núcleo de apoio à saúde da família (Nasf), no contexto da síndrome congênita por zika [Internet]. 2016[acesso em 2018 dez 10]. Disponível

em: http://189.28.128.100/dab/docs/publ icacoes/geral/estimulacao_precoce_a b.pdf

31 Teixeira MR, Couto MCV, Delgado PGG. Atenção básica e cuidado colaborativo na atenção psicossocial de crianças e adolescentes: facilitadores e barreiras. Ciênc. Saúde Colet. [Internet]. 2017[acesso em 2017 jun 13];22(6):1933-42. Disponível em: http://www.scielo.br/pdf/csc/v22n6/ 1413-8123-csc-22-06-1933.pdf

32 Gomes FMA, Cintra AMO, Ricas J, Vecchia MD. Saúde mental infantil na atenção primária à saúde: discursos de profissionais médicos. Saúde e sociedade [Internet]. 2015[acesso em 2017 fev 22];24(1):244-58. Disponível em:

http://www.scielo.br/pdf/sausoc/v24 n1/0104-1290-sausoc-24-1-0244.pdf

33 Nunes CK, Kantorski LP, Coimbra VCC. Interfaces entre serviços e ações da rede de atenção psicossocial às crianças e adolescentes. Rev. gaúch. enferm. [Internet]. 2016[acesso em 2017 fev 10];37(3):1-8. Disponível em: 


\section{NURSING \\ ANO HEALTH}

http://www.scielo.br/pdf/rgenf/v37n 3/0102-6933-rgenf-1983144720160354858.pdf

34 Silva BC, Lutterbach MGC, Arão S, Pinheiro Z. A experiência de rodas de terapia comunitária integrativa em CAPS e em ESF. Temas em educação e saúde [Internet]. 2017[acesso em 2017 fev 17];9(1):47-53. Disponível em: https: //periodicos.fclar.unesp.br/tes /article/view/9585/6345

35 Santos JRE, Silva JH, Barbosa CRPS, Laranjeira DS, Silva KSA, Nascimento JSC. Aperfeiçoando o cuidado através da terapia comunitária integrativa: promoção à saúde durante a gravidez. Revista saúde [Internet]. 2017[acesso em 2017 jun 05];11(s/n):38-9. Disponível em:

http: / / revistas.ung.br/index.php/sau de/article/view/3124/2261

36 Reis MLA, Grandesso M. O significado da capacitação em terapia comunitária integrativa na vida dos terapeutas comunitários. Temas em educação e saúde [Internet]. 2014[acesso em 2017 fev 25];10(1):89$115 . \quad$ Disponível em: https: / / periodicos.fclar.unesp.br/tes /article/view/9617/6359

37 Rocha HA, Santos AF, Reis IA, Santos MAC, Cherchiglia ML. Saúde mental na atenção básica: uma avaliação por meio da teoria da resposta ao item. Rev. saúde pública (Online). [Internet]. 2018[acesso em 2017 jan 21];52(17):1-12. Disponível em:

http: //www.scielo.br/pdf/rsp/v52/pt _0034-8910-rsp-S1518-

87872018052000051.pdf

38 Nascimento VF, Terças ACP, Hattori TY, Cabral JF, Gleriano JS, Borges AP,
ISSN 2236 - 1987

et al. Percepção de agentes comunitários de saúde sobre visita domiciliária após aperfeiçoamento em saúde da família. Rev. APS. [Internet]. 2017[acesso em 2017 jan 24];20(3):392-402. Disponível em:

https://aps.ufjf.emnuvens.com.br/ap s/article/view/2974/1121

39 Barbiani R, Nora CRD, Schaefer R. Práticas do enfermeiro no contexto da atenção básica: scoping review. Rev. latinoam. enferm. (Online). [Internet]. 2016[acesso em 2017 maio 08];24(s/n):1-12. Disponível em: http://www.scielo.br/pdf/rlae/v24/p t_0104-1169-rlae-24-02721.pdf

40 Frateschi MS, Cardoso CL. Práticas em saúde mental na atenção primária à saúde. Psico (Porto Alegre). [Internet]. 2016[acesso em 2017 mar 12];47(2):159-68. Disponível em:

http://pepsic.bvsalud.org/pdf/psico/ v47n2/08.pdf

41 Costa DO, Souza FIS, Pedroso GC, Strufaldi MWL. Transtornos mentais na gravidez e condições do recémnascido: estudo longitudinal com gestantes assistidas na atenção básica. Ciênc. Saúde Colet. [Internet]. 2018[acesso em 2017 jan 25];23(3):691-700. Disponível em:

http://www.scielo.br/pdf/csc/v23n3/ 1413-8123-csc-23-03-0691.pdf

42 Lopes CS, Abreu GA, Santos DF, Menezes PR, Carvalho KMB, Cunha CF, et al. ERICA: prevalência de transtornos mentais comuns em adolescentes brasileiros. Rev. saúde pública (Online). [Internet]. 2016[acesso em 2017 maio 14];50 Suppl:S1-14. Disponível em: http://www.scielo.br/pdf/rsp/v50s1/ 
pt_0034-8910-rsp-S01518-

87872016050006690.pdf

43 Silva PAS, Rocha SV, Santos LB, Santos CA, Amorim CR, Vilela ABA. Prevalência de transtornos mentais comuns e fatores associados entre idosos de um município do Brasil. Ciênc. Saúde Colet. [Internet]. 2018[acesso em 2017 mar 16];23(2):639-46.

Disponível em: http://www.scielo.br/pdf/csc/v23n2/ 1413-8123-csc-23-02-0639.pdf

44 Lucchese R, Oliveira AGB, Conciani ME, Marcon SR. Saúde mental no programa saúde da família: caminhos e impasses de uma trajetória necessária. Cad. Saúde Pública (Online). [Internet]. 2009[acesso em 2017 jan 06];25(9):2033-42. Disponível em: http://www.scielo.br/pdf/csp/v25n9 /17.pdf

45 Moreira MIB, Onocko-Campos RT. Ações de saúde mental na rede de atenção psicossocial pela perspectiva dos usuários. Saúde e sociedade [Internet]. 2017[acesso em 2017 mar 23];26(2):462-74. Disponível em:

http://www.scielo.br/pdf/sausoc/v26 n2/1984-0470-sausoc-26-02-00462.pdf

46 Eslabão AD, Coimbra VCC, Kantorski LP, Pinho LB, Santos EO. Rede de cuidado em saúde mental: visão dos coordenadores da estratégia saúde da família. Rev. gaúch. enferm. [Internet]. 2017[acesso em 2017 jun 11];38(1):1-8. Disponível em: http://www.scielo.br/pdf/rgenf/v38n 1/0102-6933-rgenf-1983144720170160973.pdf

47 Paes LG, Schimith MD, Righi LB, Barbosa TM. Rede de atenção em saúde
ISSN 2236 - 1987

mental na perspectiva dos coordenadores de serviços de saúde. Trab. educ. saúde. [Internet]. 2013 maio/ago [acesso em 2017 ago 12];11(2):395-409. Disponível em: http://www.scielo.br/pdf/tes/v11n2/ a08v11n2.pdf

48 Quinderé PHD, Jorge MSB, Nogueira MSL, Costa LFA, Vasconcelos MGF. Acessibilidade e resolubilidade da assistência em saúde mental: a experiência do apoio matricial. Ciênc. Saúde Colet. [Internet]. 2013[acesso em 2017 fev 05];18(7):2157-66. Disponível em:

http://www.scielo.br/pdf/csc/v18n7/ 31.pdf

49 Eslabão AD, Coimbra VCC, Kantorski LP, Cruz AG, Nunes CK, Demarco DA. Além da rede de saúde mental: entre desafios e potencialidades. Rev. pesqui. cuid. fundam. (Online). [Internet]. 2017[acesso em 2017 maio 15];9(1):85-91. Disponível em: http://seer.unirio.br/index.php/cuida dofundamental/article/view/4646/pd f_1

50 Sapag JC, Rush B, Ferris LE. Collaborative mental health services in primary care systems in Latin America: contextualized evaluation needs and opportunities. Health expectations [Internet]. 2016 [cited 2017 June 30];19(1):152-69. Available from: https://www.ncbi.nlm.nih.gov/pmc/a rticles/PMC5055227/

51 Correia VR, Barros S, Colvero LA. Saúde mental na atenção básica: prática da equipe de saúde da família. Rev. Esc. Enferm. USP. [Internet]. 2011[acesso em 2017 fev 18];45(6):1501-6. Disponível em: 


\section{NURSING AND}

http://www.scielo.br/pdf/reeusp/v45 n6/v45n6a32.pdf

52 Santos RCA, Pessoa Junior JM, Miranda FAN. Rede de atenção psicossocial: adequação dos papéis e funções desempenhados pelos profissionais. Rev. gaúch. enferm. [Internet]. 2018[acesso em 2017 jul 15];39(s/n):1-18. Disponível em: https://seer.ufrgs.br/RevistaGauchad eEnfermagem/article/view/57448/46 570

53. Ávila MB, Siniak DS. Ações de saúde mental desenvolvidas em uma estratégia de saúde da família. Rev. enferm. UFSM. [Internet]. 2017[acesso em 2017 jun 09];7(3):388-97. Disponível em:

https: / / periodicos.ufsm.br/reufsm/ar ticle/view/26237/pdf

54 Macedo JP, Abreu MM, Fontenele $M G$, Dimenstein $M$. A regionalização da saúde mental e os novos desafios da reforma psiquiátrica brasileira. Saúde e sociedade [Internet]. 2017[acesso em 2017 maio 25];26(1):155-70. Disponível em:

http://www.scielo.br/pdf/sausoc/v26 n1/1984-0470-sausoc-26-01-00155.pdf

Data de submissão: 01/05/2017

Data de aceite: 27/06/2018

Data de publicação: 18/02/2019 